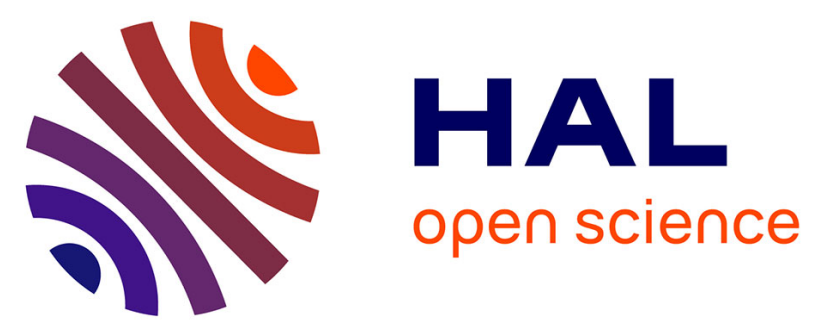

\title{
Correlation between low FAT1 expression and early affected muscle in facioscapulohumeral muscular dystrophy
}

\author{
Virginie Mariot, S. Roche, Débora Portilho, Sabrina Sacconi, Francesca \\ Puppo, Stéphanie Duguez, Philippe Rameau, Nathalie Caruso, A.L Delezoide, \\ Claude Desnuelle, et al.
}

\section{To cite this version:}

Virginie Mariot, S. Roche, Débora Portilho, Sabrina Sacconi, Francesca Puppo, et al.. Correlation between low FAT1 expression and early affected muscle in facioscapulohumeral muscular dystrophy. Annals of Neurology, 2015, 78 (3), pp.387-400. 10.1002/ana.24446 . hal-01431338

\author{
HAL Id: hal-01431338 \\ https://hal.science/hal-01431338
}

Submitted on 13 Dec 2017

HAL is a multi-disciplinary open access archive for the deposit and dissemination of scientific research documents, whether they are published or not. The documents may come from teaching and research institutions in France or abroad, or from public or private research centers.
L'archive ouverte pluridisciplinaire $\mathbf{H A L}$, est destinée au dépôt et à la diffusion de documents scientifiques de niveau recherche, publiés ou non, émanant des établissements d'enseignement et de recherche français ou étrangers, des laboratoires publics ou privés. 


\title{
Correlation between Low FAT1 Expression and Early Affected Muscle in Facioscapulohumeral Muscular Dystrophy
}

\author{
Virginie Mariot, $\mathrm{PhD},{ }^{1,2,3,4}$ Stephane Roche, $\mathrm{PhD},{ }^{5}$ Christophe Hourdé, $\mathrm{PhD},{ }^{6}$ \\ Debora Portilho, PhD, 1,2,3,4 Sabrina Sacconi, MD, PhD, ${ }^{7,8}$ Francesca Puppo, PhD, ${ }^{5}$ \\ Stephanie Duguez, PhD, 1,2,3,4 Philippe Rameau, ${ }^{9}$ Nathalie Caruso, PhD, 10 \\ Anne-Lise Delezoide, MD, ${ }^{11}$ Claude Desnuelle, MD, ${ }^{7,8}$ Bettina Bessières, MD, ${ }^{12}$ \\ Sophie Collardeau, MD, ${ }^{13}$ Leonard Feasson, MD, ${ }^{14}$ Thierry Maisonobe, MD, ${ }^{15}$ \\ Frederique Magdinier, $\mathrm{PhD},{ }^{5}$ Françoise Helmbacher, $\mathrm{PhD},{ }^{10}$ \\ Gillian Butler-Browne, PhD, 1,2,3,4 Vincent Mouly, PhD, 1,2,3,4 and \\ Julie Dumonceaux, $\operatorname{PhD}^{1,2,3,4}$
}

Objective: Facioscapulohumeral muscular dystrophy (FSHD) is linked to either contraction of D4Z4 repeats on chromosome 4 or to mutations in the SMCHD1 gene, both of which result in the aberrant expression of the transcription factor DUX4. However, it is still difficult to correlate these genotypes with the phenotypes observed in patients. Because we have recently shown that mice with disrupted Fat1 functions exhibit FSHD-like phenotypes, we have investigated the expression of the human FAT1 gene in FSHD.

Methods: We first analyzed FAT1 expression in FSHD adult muscles and determined whether FAT1 expression was driven by DUX4. We next determined FAT1 expression levels in 64 muscles isolated from 16 control fetuses. These data were further complemented with analysis of Fat1 expression in developing mouse embryos.

Results: We demonstrated that FAT1 expression is independent of DUX4. Moreover, we observed that (1) in control fetal human biopsies or in developing mouse embryos, FAT1 is expressed at lower levels in muscles that are affected at early stages of FSHD progression than in muscles that are affected later or are nonaffected; and (2) in adult muscle biopsies, FAT1 expression is lower in FSHD muscles compared to control muscles.

Interpretation: We propose a revised model for FSHD in which FAT1 levels might play a role in determining which muscles will exhibit early and late disease onset, whereas DUX4 may worsen the muscle phenotype.

View this article online at wileyonlinelibrary.com. DOI: 10.1002/ana.24446

Received Oct 28, 2014, and in revised form May 26, 2015. Accepted for publication May 26, 2015.

Address correspondence to Dr Dumonceaux, Center of Research in Myology/Institut de Myologie UMR974 — UPMC Université Paris 6/Inserm/FRE3617 — CNRS, 47, boulevard de I'hôpital, G.H. Pitié Salpétrière-Batiment Babinski, Paris Cedex 13, France. E-mail address: julie.dumonceaux@upmc.fr

From the ${ }^{1}$ Center of Research in Myology, Pierre and Marie Curie University, Sorbonne Universities, Paris; ${ }^{2}$ Mixed health research unit 974 , National Institute of Health and Medical Research, Paris; ${ }^{3}$ Unit undergoing review 3617, National Center for Scientific Research, Paris; ${ }^{4}$ nstitute of Myology, Paris;

${ }^{5}$ Timone Faculty of Medicine, Aix-Marseille University, Mixed health research unit 910, National Institute of Health and Medical Research, Marseille;

${ }^{6}$ Physiology and Exercise Laboratory, EA4338, Technolac Scientific Campus, University of Savoie Mont Blanc, Le Bourget-du-Lac; ${ }^{7}$ Mixed Unit of Research 7277, National Center for Scientific Research, Nice University Hospital, Nice; ${ }^{8}$ Neuromuscular Disease Reference Center, Nice University Hospital, Nice; ${ }^{9}$ Imaging and Cytometry Platform, Gustave Roussy Institute, Villejuif; ${ }^{10}$ Aix-Marseille University, Developmental Biology Institute of Marseille, National Center for Scientific Research Mixed Unit of Research 7288, Luminy Scientific Park, Marseille; ${ }^{11}$ Department of Developmental Biology, Robert Debré Hospital, U696, National Institute of Health and Medical Research, Paris; ${ }^{12}$ U781, National Institute of Health and Medical Research and IMAGINE Foundation, Department of Genetics, Necker Hospital for Sick Children, Public Hospital Network of Paris and Paris Descartes University, Paris; ${ }^{13}$ East Pathology Center, University Hospital Center, Lyon, Bron; ${ }^{14}$ Physiology and Exercise Laboratory EA4338, Rare Neuromuscular

Diseases Referent Center, Rhône-Alpes Bellevue Hospital, University Hospital Center of Saint-Étienne, Saint-Étienne; and ${ }^{15}$ Department of Clinical Neurophysiology, Pitié-Salpêtrière Hospital Group, Paris, France 
$\mathrm{F}$ cioscapulohumeral muscular Dystrophy (FSHD) is an autosomal dominant neuromuscular disorder (prevalence of 1:12,000-1:20,000). ${ }^{1,2}$ The pathology is characterized by (1) an asymmetric atrophy of the muscles of the face, shoulders, and arms, leading to muscle weakness ${ }^{3,4}$; and (2) some nonmuscular symptoms. ${ }^{5-8}$ The disease is caused by a loss of epigenetic marks within the $3.3 \mathrm{~kb}$ tandemly repeated sequence named D4Z4 located in the subtelomeric region of chromosome 4. This contraction leads to chromatin relaxation, and in 95\% of FSHD patients (named FSHD1), this chromatin relaxation is associated with a contraction of the D4Z4 array. ${ }^{9,10}$ The remaining 5\% of FSHD patients (named FSHD2) do not present a contraction of D4Z4, but $85 \%$ of them carry a mutation in the epigenetic modifier gene SMCHD1. ${ }^{1,11}$ This chromatin relaxation, in association with a permissive chromosome 4 , might result in an inefficient repression of the transcription factor DUX4. ${ }^{12-15}$ However, if DUX4 has been robustly associated with FSHD, ${ }^{14,16-23}$ its expression does not recapitulate the pathological features of FSHD.

Recently, we have shown that 2 distinct mutations in mice interfering with functions of the planar cell polarity Fat1 gene reproduce many of the cardinal clinical and pathological features of FSHD, including an asymmetric shoulder girdle myopathy and exudative retinopathy. ${ }^{24}$ FAT1 mRNA has been found to be specifically downregulated in several human FSHD1 fetal muscle biopsies compared to age-matched controls. ${ }^{24}$ It is therefore critical to decipher the function of FAT1 in skeletal muscle to better understand its implication in the FSHD phenotype and to identify new potential therapeutic targets for FSHD. The FAT1 protein belongs to the FAT cadherin subfamily, which is conserved across species and is among the largest cadherin molecules (for review, see Sopko and McNeill, ${ }^{25}$ Tanoue and Takeichi $\left.^{26}\right)$. Because in kidney, the human FAT1 mRNA is subject to alternative splicing events, leading to different splice isoforms, ${ }^{27}$ we investigated whether similar splice variants may occur and play distinct functions in skeletal muscle.

By analyzing FAT1 expression in human fetal muscle biopsies, we identified 2 FAT1 isoforms. Interestingly, whereas global FAT1 mRNA expression decreased with fetal development, the ratio between these 2 isoforms is conserved. Moreover, by comparing the endogenous level of FAT1 mRNA in 64 muscle biopsies isolated from 16 control fetuses, we observed lower expression levels of FAT1 in the muscles that are affected early in FSHD patients. This lower expression does not seem to be mediated by DUX4. We propose a model in which individuals with a lower level of FAT1 expression compared to the general population might be more susceptible to FSHD onset. FAT1 levels might play a role in the FSHD pathophysiology by predisposing selective subsets of muscles to an early onset of clinical symptoms and might be more sensitive to increased levels of DUX4.

\section{Materials and Methods}

\section{Biopsies}

Fetal control biopsies were isolated following therapeutic abortion from fetuses presenting diseases other than muscular pathologies. For some samples, the protocol was approved by the Biomedicine Agency (protocol number PFS12-007 and PFS13006), whereas for others, human samples and associated data were obtained from the Cardiobiotec biobank (Civil Hospices of Lyon, France). The characteristics of each biopsy are summarized in Supplementary Table 1. FSHD2 patients have been already described in other publications. ${ }^{11}$ By using sodium bisulfite sequencing, we have previously shown ${ }^{28}$ that the proximal D4Z4 region is more subjected to decreased methylation compared to the rest of the repeat. Therefore, we analyzed DNA methylation level for the different FSHD2 patients used here only for this region. The percentage of methylated $\mathrm{CpG}$ is $33 \%$ for FSHD2-78, $13 \%$ for FSHD2-83, and $21 \%$ for FSHD2-84.

\section{Cell Culture}

FSHD1 primary cells were derived from biopsies as previously described, ${ }^{23}$ and the myogenicity was determined by CD56 labeling analyzed on a Calibur flow cytometer (Becton Dickinson, Franklin Lakes, NJ). All the experiments have been performed with a minimum of $85 \%$ CD56-positive cells after enrichment on a magnetically activated cell sorting column, and cells were cultivated as previously described. ${ }^{23}$

\section{Reverse Transcriptase and Quantitative Polymerase Chain Reaction}

RNA extractions on both muscle biopsies and muscle cells were described previously. ${ }^{23}$ For both DUX4 and FAT1, reverse transcription and polymerase chain reaction (PCR) were also described previously. ${ }^{23,24}$ Quantitative PCR (qPCR) was designed according to the MIQE standards. ${ }^{29}$ qPCRs were performed in a final volume of $9 \mu \mathrm{l}$ with $0.4 \mu \mathrm{l}$ of reverse transcriptase (RT) product, $0.18 \mu \mathrm{l}$ each of forward and reverse primers $(20 \mathrm{pmol} / \mathrm{ml})$ and $4.5 \mu \mathrm{l}$ of SYBRwGreen Mastermix $\times 2$ (Roche, Basel, Switzerland). After qPCR, the PCR products were run on a $2 \%$ agarose gel and were cloned using the Topo cloning kit (Life Technologies, Carlsbad, CA) and sequenced. To determine the best reference gene, a statistical correlation was performed between the age of the fetuses and the threshold cycle $(\mathrm{Ct})$ values on at least 9 fetal biopsies for B2M, PPIA, $P O, G U S$, and HPRT1. Only B2M was validated as a reference gene for which $\mathrm{Ct}$ values are not impacted by the age of the 
fetuses. When different muscles were compared, an analysis of variance was performed to determine if the type of muscle (deltoid, quadriceps, triceps, trapezius) influences the $\mathrm{Ct}$ values for $B 2 M$. We did not observed such an effect $(p=0.51)$. For all these reasons, we decided to use $B 2 M$ as the reference gene for our qPCR analyses.

For FAT1 isoforms, $10 \mu \mathrm{l}$ of PCR products were run on a $5 \%$ acrylamide gel. The different bands were carefully cut, and DNA extraction was performed using the crush and soak method. After DNA precipitation, the different amplicons were cloned using the Topo cloning kit (Life Technologies) and sequenced with M13 primers. Primers used in this study are described in Supplementary Table 2.

\section{Sodium Bisulfite Sequencing}

Sodium bisulfite sequencing has been described previously. ${ }^{28}$ Briefly, $2 \mu \mathrm{g}$ of genomic DNA was denatured for 30 minutes at $37^{\circ} \mathrm{C}$ in $\mathrm{NaOH} 0.5 \mathrm{~N}$ and incubated overnight in a solution of sodium bisulfite $3 \mathrm{M} \mathrm{pH} 5$ and hydroquinone $10 \mathrm{mM}$. Converted DNA was purified using the Wizard DNA CleanUp kit (Promega, Madison, WI) following the manufacturer's recommendations, incubated for 15 minutes at $37^{\circ} \mathrm{C}$ in $\mathrm{NaOH} 0.3 \mathrm{~N}$ for the deamination of modified cytosine, and precipitated by ethanol for 5 hours at $-20^{\circ} \mathrm{C}$. After centrifugation, the DNA pellet was resuspended in $20 \mu \mathrm{l}$ of water and stored at $-20^{\circ} \mathrm{C}$ until use. Converted DNA was amplified using primer sets designed with Methyl Primer Express software (Applied Biosystems, Foster City, CA). To determine the methylation pattern of the FAT1 promoter region in fetal samples, we used the methyl-specific PCR method and designed 2 sets of primers able to amplify either methylated DNA (M) or unmethylated DNA (U). The sequences of these primers are indicated in Supplementary Table 3. Quantification of the band intensity was performed by Quantity One software version 4.6.9 (Bio-Rad, Hercules, CA). The band corresponding to either methylated or unmethylated DNA was quantified using the band detection and the background removal tools (average intensity). Values were normalized to the intensity of the $200 \mathrm{bp}$ molecular weight marker for gel-to-gel comparison. The sum of methylated and unmethylated DNA of each sample corresponds to $100 \%$.

\section{shRNA Constructions and Cell Transduction}

The shRNA directed against DUX4 and the production of the lentiviral vectors have been described previously. ${ }^{23}$

\section{FAT1 Antibody Generation and Immunohistochemistry}

Rabbits were injected with peptides ESCDDNGYHWDT or ESLAAPDLSKPR (underlined in Fig $1 \mathrm{~A}$ ) for FAT1- $\Delta 27$ and -27 c, respectively (ProteoGenix, Schiltigheim, France). Polyclonal antisera were harvested 3 months after injection and are called ET12 for FAT1- 427 and CR13 for FAT1-27c. Using Expasy (query against the UniProt Knowledgebase [SwissProt + TrEMBL]; http://web.expasy.org/blast/) 1 match (corresponding to FAT1) was found for the ESLAAPDLSKPR. Concerning the ET12 antibody, a perfect match was obtained with
FAT1 as expected, but a match was also found with FAT3 with $88 \%$ identity. As FAT3 is described to play a role in the interactions between neurites derived from specific subsets of neurons during development, ${ }^{30}$ a cross-reaction with muscle tissue might not occur. The antibodies were also tested on adult muscle sections, because the global level of FAT1 mRNA declined by $90 \%$ during development as compared to the adult level, and no clear labeling was visible on adult sections as expected (data not shown).

For the immunohistochemistry experiments, transverse sections $(10 \mu \mathrm{m})$ of isopentane frozen muscle biopsies were cut on a cryostat. FAT1 labelings were carried out using ET12 or CR13 antibodies. Sections were fixed in paraformaldehyde 4\%, permeabilized with 1\% Triton-X100 (Sigma, St Louis, MO), and blocked in $20 \%$ fetal bovine serum (FBS). FAT1 sera were diluted 1:20 in 2\% FBS-phosphate-buffered saline (PBS) $\mathrm{pH}$ 7.2 and incubated overnight at $4^{\circ} \mathrm{C}$. Sections were incubated for 1 hour with goat antirabbit Alexa 488 (1:300, Life Technologies) in 2\% FBS-PBS and then incubated for 5 minutes with Hoescht 33342 (1:1,000; Thermo Scientific, Waltham, MA) before being mounted on microscope slides using Dako (Carpinteria, CA) fluorescent mounting medium.

\section{PCR Detection of FAT1 Copy Number Variant in FSHD2 Patients}

In the 3 adult FSHD2 patients, the copy number variant (CNV) spanning exon 17 and intron 16 of the FAT1 gene and including a putative enhancer was analyzed by qPCR as previously published. ${ }^{24}$ The region was amplified with 3 different PCR primer couples, ${ }^{24}$ matching either exon 17 , the enhancer peak in intron 16, or exon 16 of the FAT1 genomic region. The relative amount of PCR products was normalized by the $\Delta \Delta \mathrm{Ct}$ method, using a PCR product matching an independent locus (Adora) outside the considered zone, and the geometric average of the 3 healthy control DNAs was used as the reference DNA (where all values were set to 1 ). The incidence of loss among the FSHD2 patients shown here was compared to the incidence of loss as described with the same parameters in our previous study on a group of 40 healthy individuals. ${ }^{24}$

\section{Results}

\section{In Vitro FAT1 mRNA Expression}

Because alternative splicing occurring between exons 26 and 28 in FAT1 pre-mRNA has been described in nonmuscle cells, ${ }^{27}$ we investigated the expression of splice variants in vitro on primary cultures of human myoblasts and myotubes (derived from a quadriceps muscle biopsy). After differentiation commenced, expression levels of FAT1 appeared constant for at least 5 days when primers covering either exons 2-3 or exons 24-25 were used (see Fig 1). Four abundant isoforms were detected by PCR using primers flanking exons 26 and 28 (see Supplementary Table 2): (1) a $\Delta 27$ isoform (FAT1- $\Delta 27)$, which corresponds to the direct junction between exons 26 and 28 (as described in National Center for 
FAT1_27a GAATCGTGCGATGACAATG ctcccatttggatcagagcgtactgccagagcacagtttaagagattgcta GGTATCACTGGGATACA

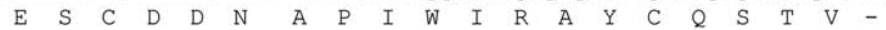

26 $27 \mathrm{c}$

FAT1_27c GAATCGTGCGATGACAATG aatctttggctgctcctgacctcagcaaaccaagag GGTATCACTGGGATACA

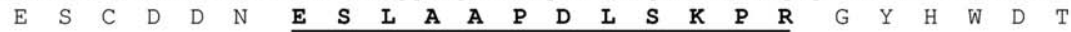
$26279 \quad 27 c \quad 28$

FAT1_27ac GAATCGTGCGATGACAATG ctcccatttggatcagagcgtactgccagagcacagtttaagagattgcta aat...gag GGTATCACTGGATACA

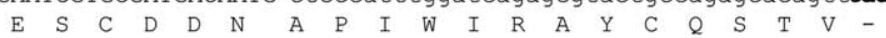
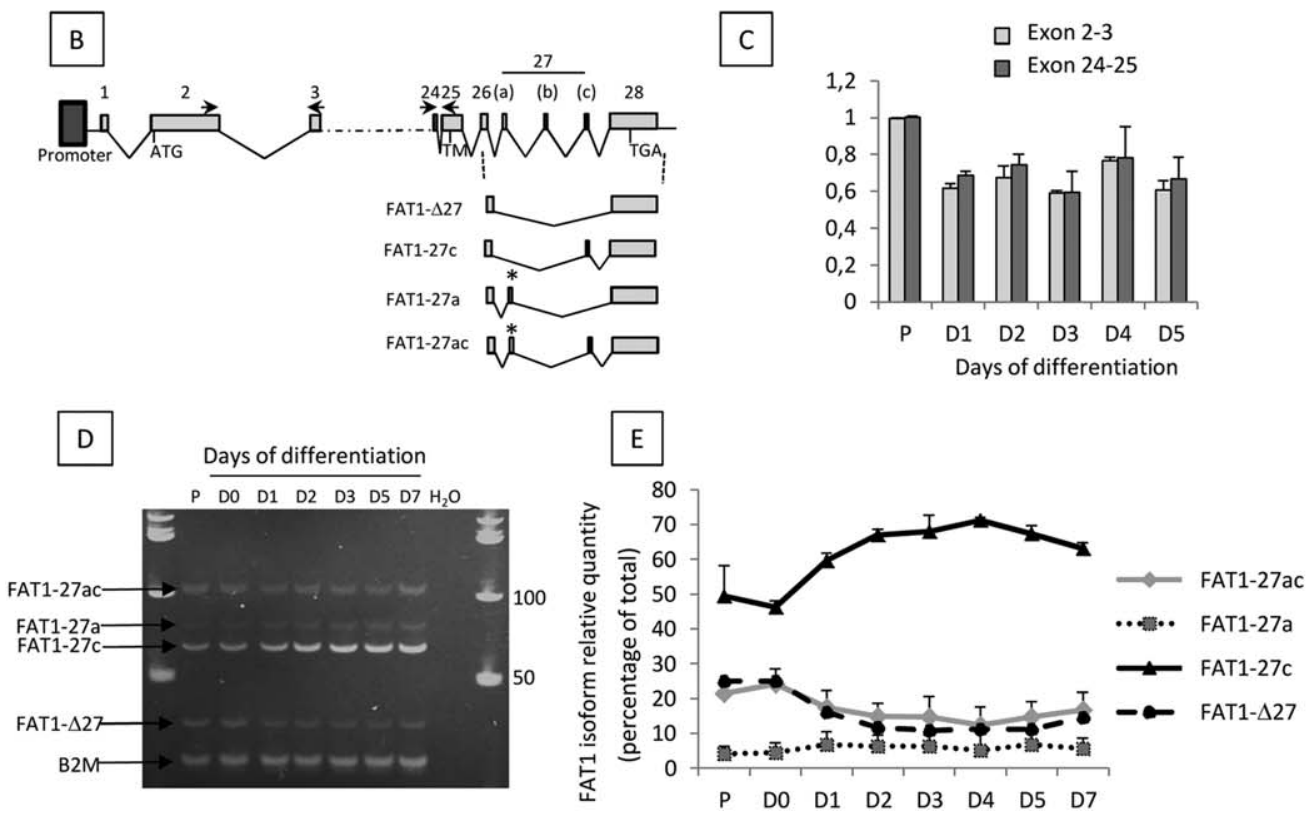

FIGURE 1: In vitro FAT1 mRNA expression. (A) Exon 26 is in black capital letters, exon 28 in gray capital letters, and alternative exons are in black lowercase letters. Stop codons are in bold. The sequences of the peptides used to generate the antibody against FAT1- $\triangle 27$ and FAT1-27c are underlined and in bold. (B) Schematic representation of the FAT1 gene. Between exons 26 and 28, 3 alternative exons have been previously reported $(+8 T R,+20$, and +12 ) and have been renamed in this article $27 a, 27 b$, and 27c, respectively. On the bottom are represented the isoforms we found in muscle cells. Asterisks indicate the presence of a premature stop codon. The primers used are indicated by arrows. TM = transmembrane. (C) A differentiation time course was performed on control primary cells. The cells were harvested at different time points, and total RNAs were extracted. Quantitative polymerase chain reactions (PCRs) on exons 2-3 and exons 24-25 were realized. An analysis of variance did not reveal any difference in FAT1 expression when primers for exons 2-3 or exons 24-25 were used $(p=0.17)$. (D, E) Primary cells were harvested at different time points after the induction of differentiation, and total RNAs were extracted. Reverse transcriptase PCR flanking exon 26-28 was carried out to amplify FAT1 isoforms between exons 26 and 28 . (D) PCR products were loaded on a $6.6 \%$ acrylamide gel. The DNA was extracted from the gel using the crush and soak method, cloned, and sequenced. (E) The relative quantity of each isoform was determined using Image J on 4 different runs during myotube formation. D0 corresponds to the day when the proliferation medium was changed for differentiation medium. The sum of intensities for the 4 isoforms was considered as $100 \%$. A multiparametric analysis of variance and a Newman-Keuls post hoc test were performed. The expression of each FAT1 isoform was statistically analyzed at 1 given day compared to the day before. For exon FAT1-27c, there was an increase in expression until D2; a statistically significant difference was observed when D0 was compared to D1 $(p=0.00014)$ and D1 to D2 $(p=0.032)$, but not when D2 was compared to D3 ( $p=0.92)$, D3 to D4 $(p=0.28)$, D4 to D5 $(p=0.36)$, or D5 to D7 $(p=0.33)$. For exons FAT1-27a and FAT1-27ac, no statistical difference was observed during differentiation. For exon FAT1- $\Delta 27$, a decrease of expression was observed between D0 to D1 ( $p=0.039$ ). The level of expression then remained stable during differentiation. $\mathrm{p}$ : probability value. $\mathrm{H}_{2} \mathrm{O}$ : without $\mathrm{RT}$ product.

Biotechnology Information CCDS47177.1); (2) a FAT1$27 \mathrm{c}$ isoform, which contains a $36 \mathrm{bp}$ fragment of intron 26 (nucleotides 5286-5321 of intron 26) included between exon 26 and 28, leading to the in frame insertion of 12 amino acids in the FAT1 protein and disturbing a putative phosphorylation site (XM_ 005262835.1);
(3) a FAT1-27a isoform containing a 51bp fragment of intron 26 (nucleotides 498-548 of intron 26), resulting in the production of a truncated protein due to the presence of a stop codon in exon 27a; and (4) a FAT1-27ac isoform, which corresponds to the inclusion of both exon $27 \mathrm{a}$ and exon 27c between exons 26 and 28, and also 
leading to a truncated protein. These results reveal the existence of 2 novel isoforms, FAT1-27a and FAT1$27 \mathrm{ac}$, raising the possibility that they may play specific roles in muscle biology.

The expression levels of these 4 isoforms evolved during differentiation (see Fig 1D, E). FAT1-27c increased as differentiation proceeded, whereas FAT1- $\Delta 27$ decreased. FAT1-27a and FAT1-27ac did not vary significantly. When evaluating the relative expression of each isoform, we found that FAT1-27c was always the major isoform and represented $49.5 \%$ of all the FAT1 RNA variants in proliferating muscle cells, whereas FAT1-27ac and FAT1$\Delta 27$ represented 21 and $25 \%$ respectively. At day 5 of differentiation, FAT1-27c represented 67\%, FAT1-27ac $15 \%$, and FAT1- $\Delta 2711 \%$, whereas expression of FAT127 a was always very low (see Fig $1 \mathrm{E}$ ).

\section{FAT1 mRNA and Protein Expression in Human Control Biopsies}

FAT1 mRNA expression levels were analyzed in 28 quadriceps biopsies isolated from control human fetuses ranging from 12 to 33 weeks of development and in 9 adult control quadriceps biopsies. Using 2 different primer sets (see Supplementary Table 2) hybridizing at the 2 extremities of FAT1 cDNA (see Fig 1A), we observed that the global level of FAT1 expression progressively declined by 90\% during fetal development (Fig 2). Linear regression reveals a correlation between FAT1 expression and fetal development $\left(R^{2}=0.199, p=0.017\right)$. Following this drastic decrease in expression, FAT1 mRNA expression levels in adult quadriceps biopsies were then very similar to what was observed at the end of fetal development, and little variation was observed during adulthood. Splicing between exons 26 and 28 was also analyzed; unlike in cultured myoblasts where 4 isoforms were detected, only 2 main isoforms (FAT1-27c and FAT1-427) were observed in vivo. The most likely explanation is that the 2 isoforms observed only in vitro could be particularly important for the primary steps of muscle differentiation (ie, during myotube formation).

To determine the subcellular localizations of these 2 protein isoforms in muscle, we raised isoform-specific rabbit polyclonal antibodies against FAT1- $\Delta 27$ and FAT1-27c (see Fig 1A). Immunohistochemistry was performed on trapezius muscle biopsies from fetal samples at 15 weeks of development (when FAT1 expression is the highest; see Fig 2D). The subcellular localizations of FAT1- $\Delta 27$ and FAT1$27 \mathrm{c}$ were very different; FAT $1-\Delta 27$ is localized within myonuclei, whereas FAT1-27c is mainly localized in the cytoplasm of muscle fibers. In the cytoplasm, FAT1-27c shows a striated pattern characteristic of the contractile apparatus, confirming our previous observations. ${ }^{24}$

\section{FAT1 mRNA Is Downregulated in Adult FSHD Biopsies}

Because we have previously shown that FAT1 mRNA is downregulated in fetal quadriceps muscle biopsies from FSHD compared to age-matched controls, ${ }^{24}$ we investigated whether such a downregulation would also occur in adult quadriceps and deltoid muscles, comparing age-matched controls and FSHD1 or FSHD2 (see Supplementary Table 1, Fig 3). A statistically significant lower (1.96-fold) expression of FAT1 was observed in the FSHD1 deltoid biopsies compared to controls. In the quadriceps, a 1.5-fold decrease in FAT1 mRNA level was observed in both FSHD1 and FSHD2 biopsies but was statistically different only for FSHD2, consistent with a high variability among FSDH1 samples (as shown by the increased standard deviation). As the quadriceps is not always clinically affected in FSHD patients even at advanced stages, we investigated whether this increased variability was correlated with the extent of the weakness in specific muscles. A clinical diagnosis was available for 4 of the patients from whom quadriceps biopsies were available, 2 of them being clinically affected whereas the 2 others were not. When the expression level of FAT1 was compared in these 4 biopsies, a 2-fold decrease was observed in the clinically affected biopsies compared to the non-clinically affected muscles. These results suggest that lower levels of FAT1 expression may be correlated with advanced disease severity. The relative abundance of each isoform was also compared in control and FSHD1 biopsies for quadriceps and deltoid muscle biopsies, and no significant difference was observed. In conclusion, in adult biopsies, the expression level of FAT1 is downregulated in FSHD1 and FSHD2 muscles without causing any significant switch in isoform usage.

As we previously observed that contractionindependent FSHD cases could carry a deletion of an intronic regulatory element of FAT1, quantification by qPCR was performed on genomic DNA isolated from muscle biopsies of FSHD2 patients (Fig 4A). Small deletions spanning the intronic FAT1 enhancer were observed, extending our previous findings, and reinforcing the link between FAT1 deregulation and presence of this CNV. The incidence of loss matching the position of exon 16 is significantly higher among FSHD2 patients (3 of 3 ) than in the group of 40 healthy individuals characterized in our previous study ${ }^{24}$ (see Fig 4B, Fischer test, $p<0.0042$ ).

\section{Muscles with Early Onset FSHD Symptoms Exhibit Lower Levels of FAT1 Expression}

FSHD is known to affect preferentially specific muscles while sparing others. We therefore investigated the relative expression levels of FAT1 in different control muscle groups. We compared FAT1 expression levels in 64 


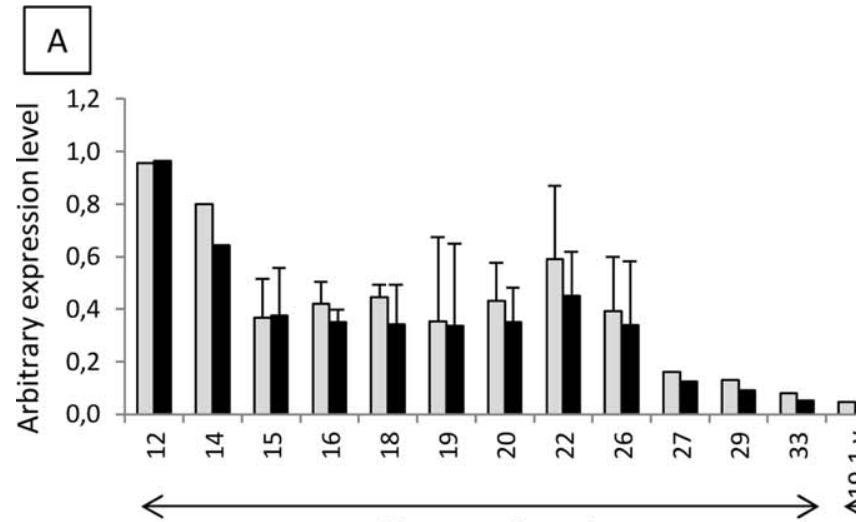

Fetal biopsies $(\mathrm{N}=28)$
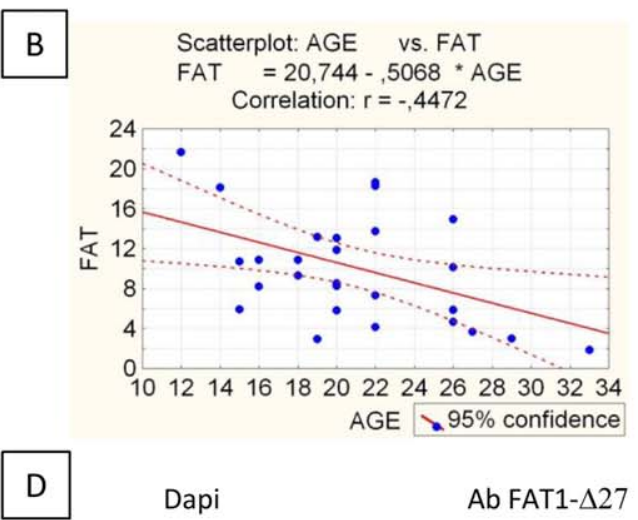

Dapi

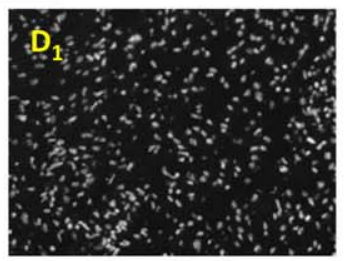

Dapi

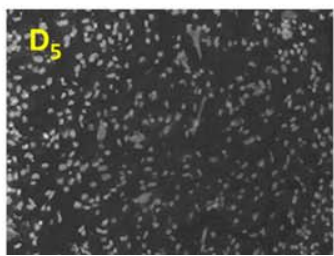

Ab FAT1- $\Delta 27$

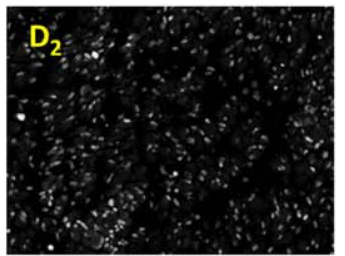

Ab FAT1-27c

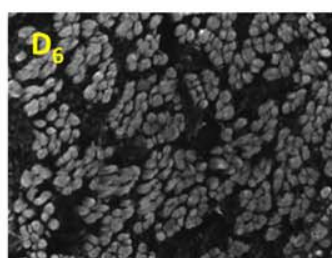

$\square 2-3$

- 24-25
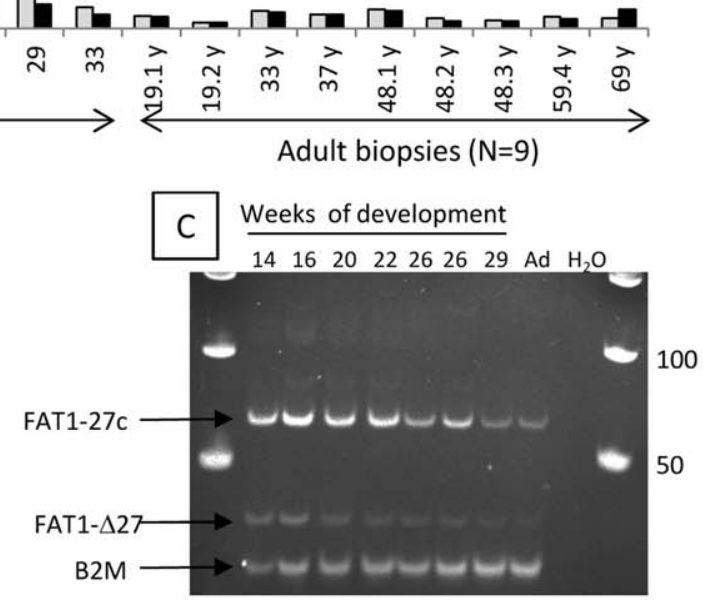

Adult biopsies $(\mathrm{N}=9)$
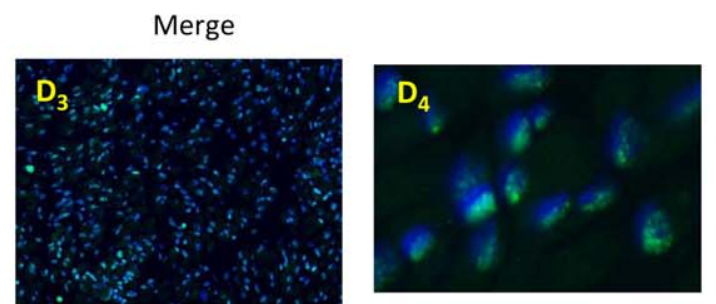

Merge
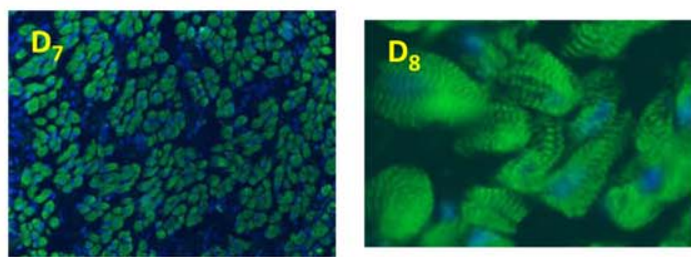

FIGURE 2: In vivo FAT1 expression. (A) Total RNAs were extracted from 28 control quadriceps biopsies from fetuses aborted at 12 to 33 weeks of development and from 9 adult control quadriceps adult biopsies. Two reverse transcriptase polymerase chain reactions (PCRs) with primers spanning either exons 2-3 or exons 24-25 were performed. B2M was used as the normalizer. The standard deviations were calculated on $n=2$ biopsies for fetuses at 15, 16, 18, and 19 weeks of development, $n=5$ biopsies for fetuses at 20 weeks of development, $n=6$ biopsies for fetuses at 22 weeks of development, and $n=6$ biopsies for fetuses at 26 weeks of development. (B) Correlation between FAT1 expression and time of development ( $n=28$ biopsies; $R^{2}=0.199, p=0.017$ ). (C) Total RNAs were extracted from control quadriceps fetal biopsies. Primers flanking exons $26-28$ were used to amplify FAT1 isoforms between exons 26 and 28, and PCR products were loaded on a $6.6 \%$ acrylamide gel. The ages of the fetuses are indicated. Ad=Adult. B2M was used as the reference gene. (D) Subcellular localization of FAT1-27c and FAT1- $\Delta 27$ on fetal control biopsies. Transverse $10 \mu \mathrm{m}$ frozen sections of either fetal trapezius muscle biopsies were performed using a cryostat. Sections were stained with rabbit polyclonal antibodies directed against FAT1- $\Delta 27$ (D2, D3, D4) or FAT1-27c (D6, D7, D8) and counterstained with 4',6-diamidino-2-phenylindole (Dapi; D1, D5). D4 and D8 represent higher magnification of D3 and D7, respectively. Scale bars: $D_{1-3}$ and $D_{5-7}=50 \mu \mathrm{m} ; D_{4}$ and $D_{8}=10 \mu \mathrm{m}$.

muscles isolated from 16 control fetuses. On each fetus, 4 muscles were isolated; 2 are usually described to be affected early in FSHD patients (trapezius and biceps), whereas the other 2 are affected late (quadriceps and deltoid). ${ }^{31}$ FAT1 expression level was found differentially expressed between these potentially early and late affected 

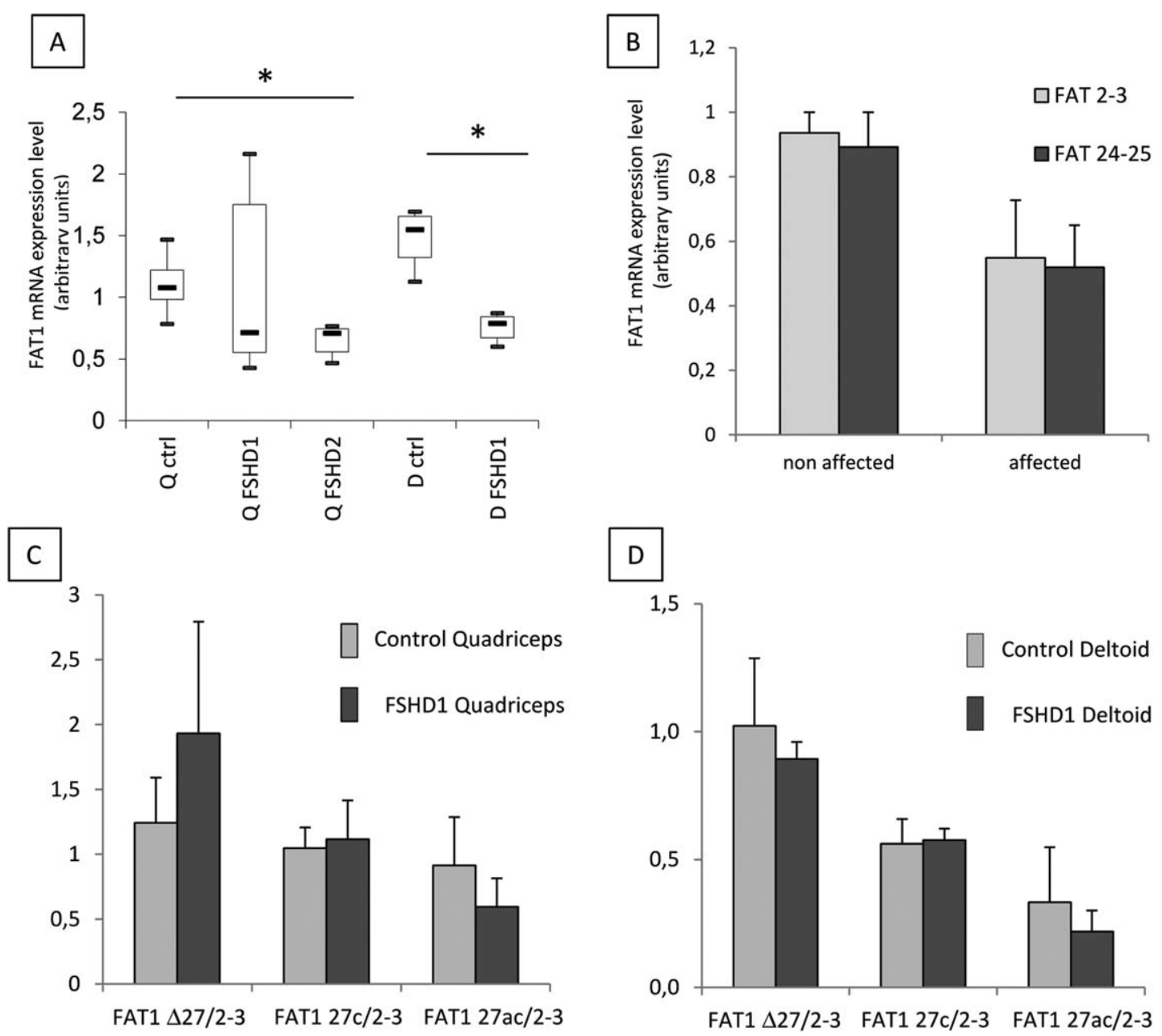

FIGURE 3: FAT1 expression in facioscapulohumeral muscular dystrophy (FSHD) biopsies. (A) FAT1 expression level was determined by quantitative polymerase chain reaction on control and FSHD biopsies. The box plots correspond to B2M normalized data. Experiments were made in triplicate. Horizontal lines are medians, the extremities of the boxes are delimitated by the first and third quartile, and the whiskers correspond to the 10th and 90th percentile. A Wilcoxon-Mann-Whitney statistical analysis was performed. ${ }^{*} p<0.05$. The experiments were performed in triplicate on $n=9$ control (ctrl) quadriceps (Q) biopsies, $n=7$ FSHD1 quadriceps biopsies, $n=3$ FSHD2 quadriceps biopsies, $n=5$ control deltoid (D) biopsies, and $n=3$ FSHD1 deltoid biopsies. (B) The expressions of FAT1 were analyzed with primers spanning exon 2-3 and exon 24-25 on 2 clinically affected and 2 non-clinically affected quadriceps muscle biopsies. (C, D) The expression levels of each FAT1 isoform were analyzed in quadriceps (C) or deltoid (D) control and FSHD1 biopsies. To avoid intrinsic variations linked with internal FAT1 exon 2-3 transcription, the expression level of each isoform was normalized to FAT1 exon 2-3. B2M was used as a normalizer in all experiments.

muscles $(p<0.05$; Fig $5 \mathrm{~A})$. Interestingly, the expression levels of FAT1 were lower in the muscles with early onset symptoms in FSHD compared to muscles affected at later stages (respectively by $38 \%$ and $28 \%$ for the trapezius and biceps compared to quadriceps). Statistical analyses demonstrated that this difference in FAT1 expression levels between early and late affected muscles is independent of the developmental age of the fetuses, and a linear regression reveals a correlation between FATI expression and early affected muscles $\left(R^{2}=0.13\right.$, $p=0.0046)$. To determine whether this lower FAT1 expression level in late affected muscle could be linked to an increased DNA methylation at the FAT1 promoter, a methyl-specific PCR amplification was performed after sodium bisulfite modification on DNA from biceps, trapezius, deltoid, and quadriceps biopsies from 3 fetuses. This analysis did not reveal any significant difference in the DNA methylation level between the different muscle biopsies (see Fig 5B, C), thus suggesting that differential FAT1 levels are not regulated by methylation changes at the FAT1 promoter in the different muscles.

These results suggest that, although FAT1 expression level progressively decreases during fetal development, muscles that represent early targets in FSHD express lower levels of FAT1 as compared to muscles that are later targets. Consistent with this observation, we also 
observed in E13.5 Fat1 ${ }^{\text {LacZ/+ }}$ mouse embryos, in which LacZ reporter expression recapitulates the endogenous domain of Fat 1 expression, ${ }^{24}$ that most of the muscles known to be affected at early age of FSHD in humans exhibit low levels of $\beta$-galactosidase activity in mice, whereas muscles affected at later disease stages or not affected exhibit higher $\beta$-galactosidase activity (see Fig $5 \mathrm{D}, \mathrm{E}$ ), indicating an inverse correlation between Fat1 expression levels and the stage of disease onset in FSHD. Some muscles however do not comply with this rule, such as the triceps brachii or the rhomboid superior muscles, both of which express high levels of Fat ${ }^{\text {LacZ }}$
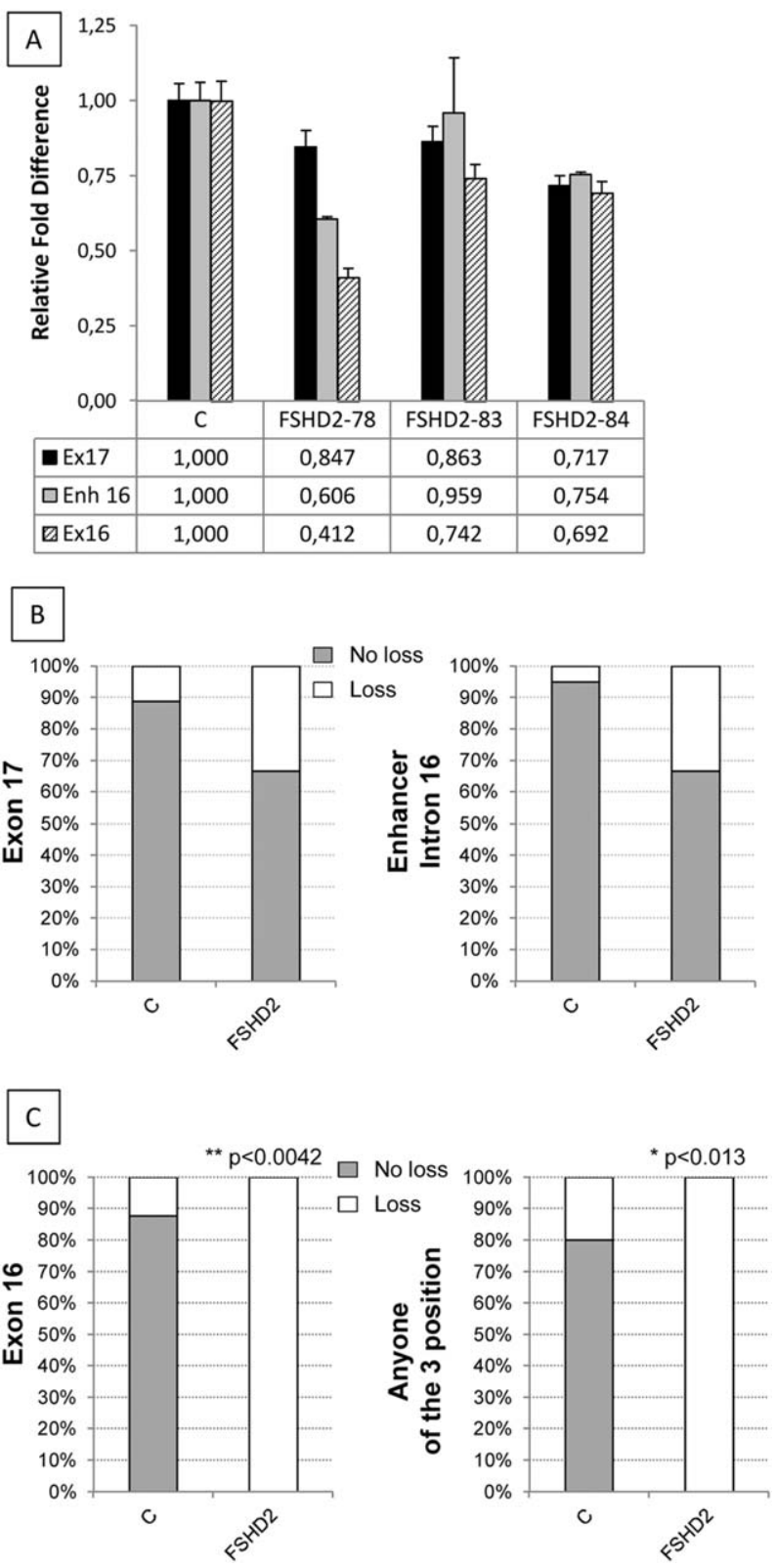

FIGURE 4: despite being affected at early disease stages in FSHD (see Fig 5E).

\section{DUX4 Is Not Responsible for FAT1 Downregulation in FSHD Myotubes}

Because DUX4 is a transcription factor previously described to modulate the expression of hundreds of genes, and because we showed that when overexpressed in human myoblasts DUX4 was capable of repressing FAT1 expression by 4 -fold, ${ }^{24}$ we next investigated a possible role of DUX4 in the regulation of FAT1 in FSHD cells. Four adult FSHD primary cultures were transduced with a lentiviral vector encoding an shRNA against all DUX4 mRNA isoforms (shDUX4) or with an empty shRNA. DUX4 expression was analyzed after 3 and 4 days of differentiation, and we confirmed that DUX4 mRNA was reduced in the presence of the shDUX4 (Fig 6). Accordingly, the expression levels of ZSCAN4 and $M B D 3 L 2,2$ genes downstream of the DUX4 cascade, were decreased by 2.3- to 2.8-fold and 2.6- to 3-fold at day 3 and 4, respectively. However, the downregulation of DUX4 did not modify the levels of expression of either the full-length or alternative FAT1 transcripts, suggesting that the downregulation of FAT1 in FSHD is not

FIGURE 4: FAT1 copy number variant (CNV) in facioscapulohumeral muscular dystrophy (FSHD)-2. (A) Analysis of deletion of the intronic FAT1 enhancer. The graph represents the relative amounts of polymerase chain reaction (PCR) fragments obtained using primers around exon (Ex) 17 (black bars), the enhancer (Enh) in intron 16 (gray bars), and the exon 16 (hatched bars) of the FAT1 gene in 3 healthy controls (average taken as the reference and represented as C) and the 3 adult FSHD2 patients. All data were normalized by the $\Delta \Delta \mathrm{Ct}$ method using an unrelated genomic fragment (Adora) as internal control, and the geometric average of the 3 healthy control DNAs was used as the reference DNA (where all values are set to 1). Error bars represent confidence interval for each average fold-value obtained $(n=3)$. A cutoff of 0.75 has been set. Individuals in whom the relative value is lower than the cutoff are considered as having lowered copy numbers (indicated as loss). (B, C) Incidence of FAT1 CNV among the 3 FSHD2 patients studied, compared to controls (C) from Caruso et al. ${ }^{24}$ For each position studied by quantitative PCR (exon 17, enhancer in intron 16, and exon 16), when the relative copy number is below the cutoff of 0.75 , the corresponding individual is considered as carrying a loss (low copy numbers). Incidence is represented as percentage of individuals carrying loss (white) or without loss (gray) for the group of 3 FSHD2 patients studied and for the control group of 40 healthy individuals from our previous study (these are the same incidence results as those reported in Caruso et al, ${ }^{24}$ obtained through identical experiments, the reference control individuals being the same). The bottom graph represents the incidence of loss at any of the 3 points studied. In B, there were too few affected individuals to do statistical testing; in C there were too few for chi-square testing, but probability values for Fisher test are indicated. 


\section{A}

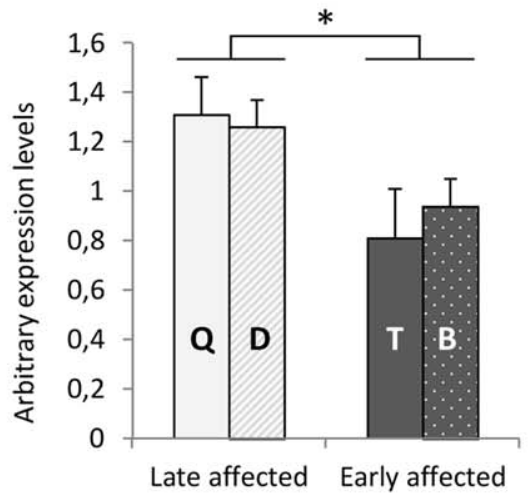

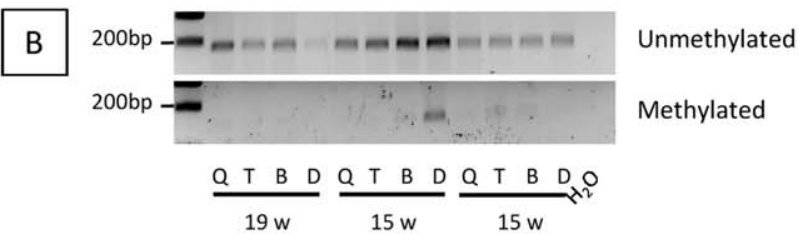
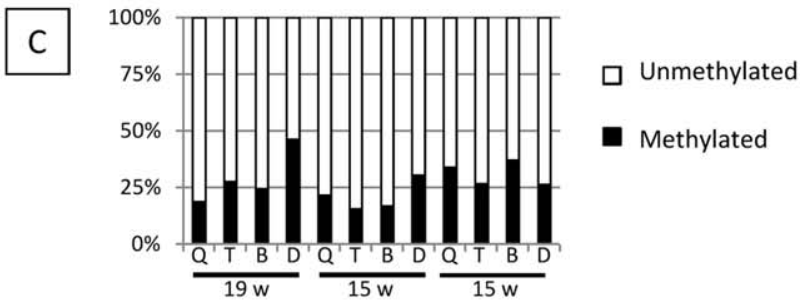
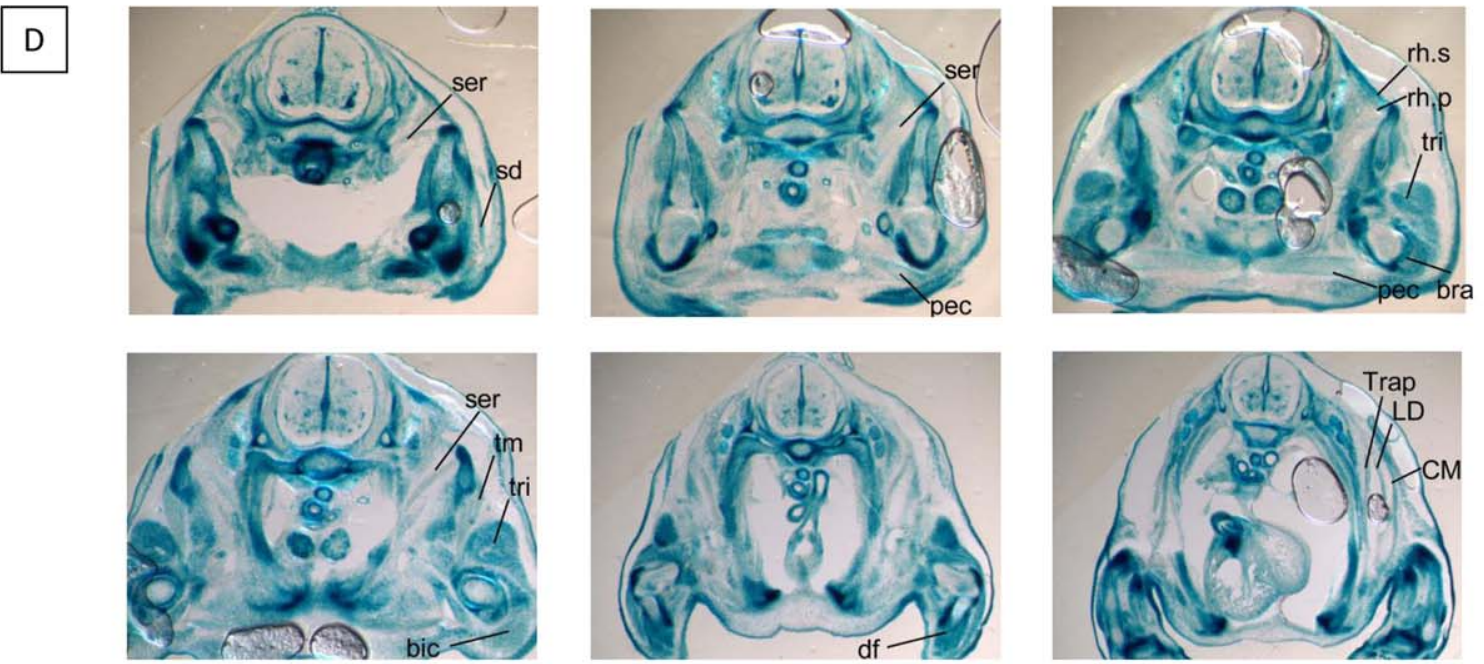

\begin{tabular}{|c|c|c}
\hline Muscle mouse name & $\beta$-Galactosidase levels & Age of onset of FSHD symptoms \\
\hline Serratus ant. (ser) & low & early \\
\hline Pectoralis major (pec) & low & early \\
Biceps Brachii Long. (bic) & low & early \\
Teres major (tm) & low & early \\
Cutaneous maximus (cm) & low & not applicable \\
\hline Lassimus Dorsi (LD) & low & early \\
Trapezius (trap) & low & early \\
Rhomboid prof (rh. P) & low & early \\
Rhomboid Sup (rh. S) & medium & late \\
Spinodeltoid (sd) & medium & late \\
Triceps Brachii long. (tri) & high & not clear \\
Distal Forelimb (df) & high & not affected \\
Brachialis (bra) & high & ??
\end{tabular}

FIGURE 5: FAT1 expression in early and late affected muscles. (A) Total RNAs were extracted from 16 control fetuses. On each fetus, quadriceps (Q), trapezius (T), biceps (B), and deltoid (D) were analyzed for FAT1 mRNA level using primers covering exons 2-3. A multiparametric analysis of variance and a Newman-Keuls post hoc test were performed on early and late affected muscles. ${ }^{\star} p<0.05$. A linear regression reveals a correlation between FAT1 expression and early affected muscles $\left(R^{2}=0.13, p=0.0046\right)$. (B, C) The methylation status of the FAT1 promoter was analyzed by methylation-specific polymerase chain reaction amplification after sodium bisulfite modification on fetal control quadriceps, trapezius, biceps, and deltoid biopsies. $C$ represents the percentage of methylated (black) or unmethylated (white) CpG. (D) Skeletal muscle groups were visualized at E14.5 in Fat1 $1^{\text {LacZ/+ }}$ mouse embryos by X-gal staining. Muscle names are indicated. (E) Comparison of $\beta$-galactosidase expression levels in Fat1 $1^{\mathrm{LacZ} /+}$ mouse embryos and the age of onset of facioscapulohumeral muscular dystrophy (FSHD) patients. The muscles we analyzed are located in the shoulder and forearm area (which are not spared in FSHD), and we have distinguished those muscles involved earlier or later in FSHD. 

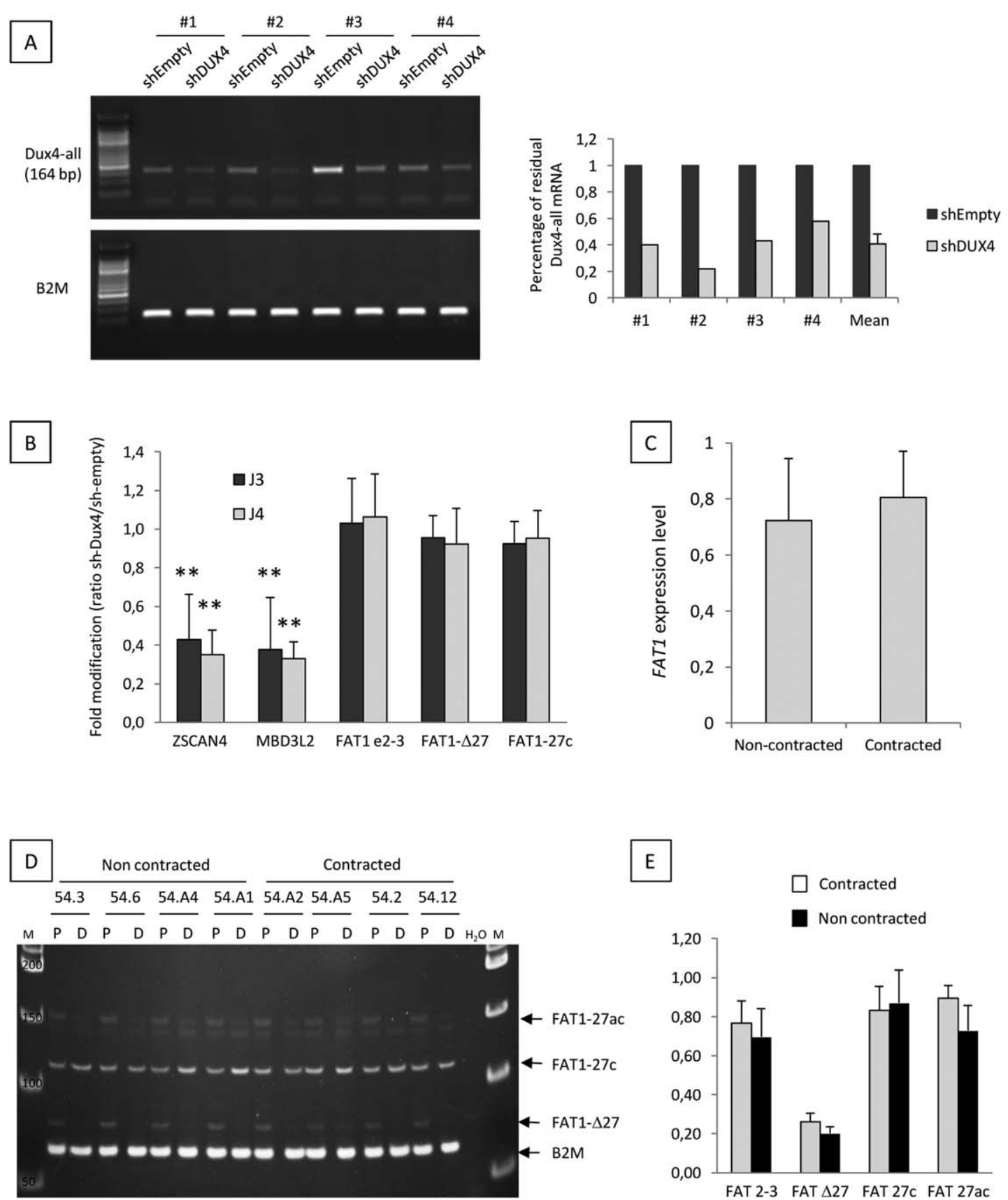

FIGURE 6: Link between DUX4 and FAT1 expressions. (A) Primary cells were stably transduced using either an shRNA against DUX4 (shDUX4) or an empty shRNA. Cells were harvested at day 3 and 4 after induction of differentiation. DUX4 expression levels were determined by polymerase chain reaction (PCR) and run on a $2 \%$ agarose gel (left panel). The percentage of residual DUX4-all mRNA was calculated after quantitative PCR (qPCR; right panel). (B) The expression levels of FAT1 and of 2 genes downstream of DUX4 were determined by qPCR at day 3 or 4 after induction of differentiation. The graph represents the ratio shDUX4/shEmpty. (C) Using primers spanning exon 2-3, FAT1 expression levels were analyzed in differentiated myotubes (day 5) from contracted or noncontracted clones isolated from 1 mosaic patient. (D, E) Immortalized facioscapulohumeral muscular dystrophy clones were harvested either in proliferation (P) or at day 4 of differentiation (D), and total RNAs were extracted. Reverse transcriptase PCR flanking exons 26-28 was realized to amplify FAT1 isoforms between exon 26 and 28 . PCR products were run on a $5 \%$ acrylamide gel (D). The relative quantity of each isoform was determined by qPCR (E). In all of these experiments, B2M was used as the reference gene. ${ }^{\star *}: p<0.01, M: 50$ bp ladder.

mediated by DUX4. This result was confirmed by analyzing FAT1 expression levels in contracted and noncontracted immortalized clones isolated from an FSHD1 mosaic patient. ${ }^{32}$ These clones present an identical geno- type except for the presence or absence of the D4Z4 repeat contractions, and DUX4 mRNA was only detected in the contracted clones. In these cell cultures, no difference was observed in FAT1 level between contracted and 


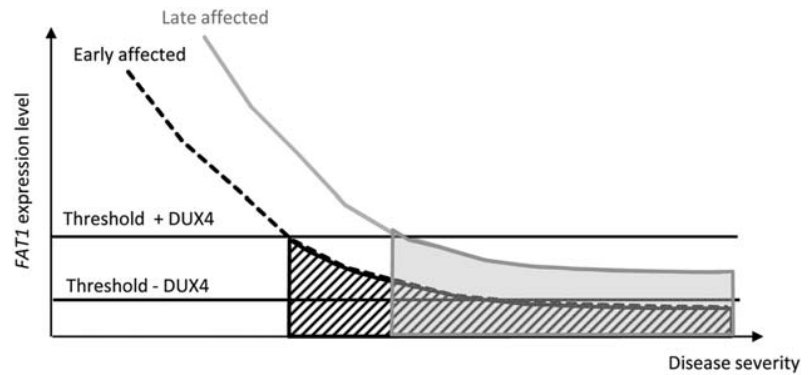

FIGURE 7: Schematic diagram of the FAT1 contribution in facioscapulohumeral muscular dystrophy (FSHD onset in muscle biopsies. The $y$-axis represents the disease severity, whereas the $x$-axis represents FAT1 expression levels. Early affected muscles are represented by a dotted black line and late affected muscles by a gray line. Hatched and gray areas correspond to the onset of symptoms of early and late affected muscles, respectively. In this model, FAT1 level determines the patterning of early affected muscles, and the factor that determines whether a group of muscles will be affected early or late in FSHD could be a critical threshold in FAT1 expression level. When the level of FAT1 is high (for deltoid and quadriceps), the muscles are spared for a longer time, but when the level of FAT1 is low (for trapezius and biceps), the muscles are more sensitive to gene deregulations. This threshold could be DUX4 dependent or DUX4 independent.

noncontracted clones, whereas only the contracted clones express DUX4 mRNA. ${ }^{32}$ Moreover, no difference in the expression of FAT1 variants was observed in the contracted clones compared to the noncontracted clones. This once again argues against the possibility that reduced FAT1 expression might have been caused by DUX4.

A reverse role of FAT1 in the regulation of expression of DUX4 was also investigated. Control primary cultures (4qA haplotype) were transduced with lentiviral vectors expressing an shRNA directed either against all FAT1 isoforms (FAT1 exon 2-3) or specifically against each isoform. Because some isoforms share sequences, the shRNAs were carefully designed to target only 1 specific mRNA isoform of FAT1. All the shRNAs were functional and induced a downregulation of the targeted FAT1 isoform, but DUX4 mRNA was not detected in any of the samples, despite their 4qA haplotype, suggesting that downregulation of FAT1 is not sufficient to promote the increase in DUX4 expression (data not shown).

\section{Discussion}

\section{FAT1 Expression in Control Muscle Biopsies}

We have shown in fetal control biopsies a strong correlation between low levels of FAT1 expression and muscles that are affected at early disease stages in FSHD. This model is supported by our analysis of Fat ${ }^{\text {LacZ }}$ expression in developing mouse embryos, in which a large subset of scapulohumeral muscles, known to belong to the early FSHD map, express lower levels of Fat ${ }^{\text {LacZ }}$ than muscles affected at later stages of disease progression or not affected. The few exceptions to this correlation suggest that there are other components to the FSHD map. The biological significance of these different expression levels of FAT1 in different muscles remains to be elucidated, and what determines how FAT1 is regulated in these different muscles is still unknown. Our analysis of FAT1 promoter methylation already shows that no distinction can be made between muscles. Furthermore, the map of muscles with low FAT1 expression and predicted early onset symptoms does not simply match with 1 embryonic territory with unique molecular origins, but instead encompasses several such domains.

\section{Reduced FAT1 Levels in FSHD Biopsies}

Recently, we have shown a decreased expression of FAT1 mRNA in FSHD fetal muscle biopsies as compared to age-matched controls, ${ }^{24}$ suggesting a participation of FAT1 in the pathophysiology of FSHD. Here, we found that such a decreased expression is maintained in adult muscle, as we observed a 1.96-fold reduction in FAT1 expression levels in adult FSHD1 deltoid biopsies compared to control deltoid biopsies. Understanding the mechanism that could lead to this reduction of FAT1 expression will provide important insight into FSHD pathogenesis. We first investigated the possible role of DUX4 because DUX4 is a transcription factor regulating hundreds of mRNAs and is upregulated in FSHD muscle biopsies. $^{20,33-35}$ However, we were unable to demonstrate a link between FAT1 and DUX4 expression. This result contrasts with the previous finding that experimental overexpression of exogenous DUX4 in human cells causes a decrease of FAT1 expression after 24 hours. $^{20,24,33}$ Although DUX4 may be capable of silencing FAT1 expression, our experiments show that DUX4 expression (in conditions where DUX4 overexpression is not experimentally induced) is not directly responsible for the decrease in FAT1 expression. In the context of FSHD, DUX4 silencing was not shown to alter FAT1 expression, supporting the idea of an absence of regulation of FAT1 by DUX4. This possibility is supported by our finding that there were no differences in FAT1 expression levels between contracted and noncontracted clones isolated from a mosaic patient, confirming that FAT1 levels are not correlated to DUX4 expression.

One possible mechanism by which FAT1 expression is decreased in FSHD muscles is provided by our previous identification of a CNV deleting an intragenic putative regulatory enhancer in the FAT1 locus, which we found to segregate with FSHD. ${ }^{24}$ Such deletions are 
predicted to cause tissue-specific changes in FAT1 expression. Although insufficient when heterozygous to cause FSHD on its own (as a small percentage of healthy individuals can carry this $\mathrm{CNV}$ ), such $\mathrm{CNV}$ was found to be significantly enriched in a group of FSHD patients without canonical D4Z4 contraction, as well as in a small group of FSHD1 patients, ${ }^{24}$ suggesting that it may segregate and cooperate with DUX4. Interestingly, among the 3 FSHD2 cases used in the present study, in which reduced levels of FAT1 expression were found in quadriceps muscles, 2 were found to carry the described $\mathrm{CNV}$ in $F A T 1^{24}$ and all 3 of them carried a loss at the level of exon 16. Such deletions could alter the tissue-specific distribution of FAT1 expression.

\section{Relationship between FAT1 and FSHD}

Low levels of FAT1 expression were observed in 2 independent situations: (1) in control fetal human biopsies or in developing mouse embryos, FAT1 is expressed at lower levels in muscles usually described as affected at early stages of FSHD progression than in muscles described as affected later or as nonaffected; and (2) in adult muscle biopsies, FAT1 expression was lower in FSHD1 and FSHD2 muscles, compared to control muscles. These observations suggest that lower or decreased levels of FAT1 (whether naturally low as specified by the developmental program or additionally reduced in an FSHD-related context) may sensitize the muscle to the cascade of gene deregulations caused by the $\mathrm{D} 4 \mathrm{Z} 4$ repeat contraction. Thus, according to such a model, individuals with an intrinsically low FAT1 expression level may be more susceptible to FSHD onset. This scenario is further supported by the observation in the Fat1${ }^{\text {LacZ/+ }}$ mouse embryos that muscles exhibiting low levels of $\beta$-galactosidase activity match in most cases with the muscles with early onset symptoms in FSHD in humans. Furthermore, this correlative map also matches the selective map of scapulohumeral muscles exhibiting wasting in adult Fat ${ }^{\text {LacZILacZ }}$ mice, ${ }^{24}$ although some of these early onset muscles (rhomboid, triceps) do exhibit high levels of Fat1 expression at the stage analyzed. The similarity between the map of muscles exhibiting embryonic or adult defects in Fat1-deficient mice, and the map of muscles affected in FSHD patients strongly argues in favor of a direct participation of FAT1 in FSHD onset. Which FAT1 isoform is the most relevant isoform, the loss of which could contribute to this muscle deterioration, remains to be determined, because FAT1-27c and FAT1- $\Delta 27$ have different subcellular localizations and may play different functions.

\section{A Revised Model for FSHD Onset}

Altogether, these results allowed us to propose a new model for FSHD onset that complements recent findings showing that DUX4-induced gene expression is the major molecular signature in FSHD skeletal muscles. ${ }^{34}$ In this revised model, FAT1 determines the patterning of early affected muscles (Fig 7). FAT1 and DUX4 do not regulate each other but both may participate independently to the muscle phenotype. We have previously proposed that because DUX4 is expressed during fetal development, whereas the clinical signs of the pathology appear 15 to 20 years later, a second event must be required. ${ }^{23}$ This event could be the intrinsic FATI expression levels in each muscle. We propose that what determines whether a group of muscles will be affected early or late in FSHD is the FAT1 threshold below which muscles will be affected. When the level of FAT1 is high, the muscles are spared for a long time, whereas when the FAT1 level is low, the muscles are more sensitive to gene deregulations caused by the $\mathrm{D} 4 \mathrm{Z} 4$ deletions and become affected earlier. This threshold could be DUX4 dependent or DUX4 independent and is supported by the recent discovery of FSHD-like patients with neither a D4Z4 contraction, nor an SMCHD1 mutation but with mutations in FAT1. ${ }^{36}$ In the presence of DUX4, the critical threshold might be higher than in the absence of DUX4. Individuals with very low levels of FAT1 and for whom FSHD might be DUX4independent are probably very rare because transgenic Fat $1^{-/-}$mice exhibit perinatal lethality probably due to loss of the renal glomerular defects and in some cases defects in forebrain development. ${ }^{37}$ In drosophila, FAT depletion causes defects in differentiation and morphogenesis, and lethality at the pupal stage, ${ }^{38}$ indicating the importance of FAT in organ development. However, rare cases of FSHD patients carrying the nonpermissive 4B163 haplotype (which does not carry the DUX4 poly[A] signal) have been described, ${ }^{39}$ suggesting that FSHD could occur in the absence of DUX4. A DUX4independent form of FSHD is also supported by the mouse model disturbing Fat1, which develops a phenotype mimicking FSHD in the absence of DUX4 expression. ${ }^{24}$

In conclusion, both high DUX4 and low FAT1 expression levels may be essential for triggering the onset of FSHD. Due to chromatin relaxation, DUX4 is expressed in some FSHD muscles since fetal development $^{22,23}$ and may slowly worsen muscle and muscles expressing low levels of FAT1 are the first to be affected. The proximity of FAT1 and the D4Z4 array on chromosome 4 is interesting, because it indicates that any genetic variant occurring in FAT1 will have a high probability of cosegregating with a permissive/pathogenic $4 \mathrm{q} 35$ haplotype and vice versa. Second, besides the DNA alterations occurring at regulatory sequences of the FAT1 gene, 
which have the potential to deregulate FAT1 expression, the changes occurring around the D4Z4 array may indirectly cause deregulation of FAT1 expression, either in cis or in trans. Although we rule out a contribution of DUX4 overexpression to the lowered FAT1 levels observed in FSHD myoblasts, other mechanisms may involve the long noncoding RNA DBE-T, ${ }^{40}$ or altered interaction with the nuclear membrane, hence indirectly affecting the level of FAT1 expression.

Several questions are still pending, including the following. When does FAT1 expression level trigger FSHD? Is it during fetal development, after birth, or both? How does FAT1 expression participate to muscle decline? FAT1 is a cadherin protein ${ }^{26,41}$ and may be very important during the early stages of fetal muscle development when a high level of expression is required. In addition, FAT1 protein is detected in skeletal muscles in close proximity to the voltage-dependent calcium channel dihydropyridine receptor, ${ }^{24}$ which would suggest a direct role of FAT1 in muscle biology.

\section{Acknowledgment}

This study was financially supported by the French Association against Myopathies (AFM-Téléthon, France), Pierre and Marie Curie University Emergence 2010 (V.Ma.), the FSHD Global Research Foundation (grant 14), the National Research Agency (FSHDecrypt, ANR-09-GENO038 and FSHDecipher, ANR-13-BSV1-0004), a fellowship from the FSH Society (FSHS-22012-03) (V.Ma.), and CNPq-Inserm French-Brazilian International Laboratory of Cell Therapy and Immunotherapy (490272/2008-8; D.P.).

Human samples were obtained from the Cardiobiotec biobank (Civil Hospices of Lyon, France; authorized by the French Ministry of Social Affairs and Health as DC2008-139 with cession authorization AC 2008-113), the Myobank-AFM tissue bank, the fetopathologists of Marseille, and the SOFFOET (Société Française de Foetopathologie) according to protocol numbers PFS12-007 and PFS13-006, approved by the French Agency of Biomedicine of the Ministry of Health.

We thank all the patients who provided the biopsies; and S. Denadai and the Astre and Anabiomol platforms of the University of Savoie Mont Blanc for help in histology.

\section{Authorship}

Conceived and designed the experiments: J.D., V.Ma., F.H., F.M. Performed the experiments: V.Ma., S.R., C.H. D.P., F.P., P.RN.C. Contributed reagents/materials/analysis tools: C.H., S.S., F.M., A.-L.D., C.D., B.B., S.C.,
L.F., T.M., F.H., G.B.-B., S.D., V.Mo., J.D. Wrote the article: G.B.-B., V.Mo., F.M., F.H., J.D.

\section{Potential Conflicts of Interest}

Nothing to report.

\section{References}

1. Tawil R, van der Maarel SM, Tapscott SJ. Facioscapulohumeral dystrophy: the path to consensus on pathophysiology. Skelet Muscle 2014;4:12.

2. Deenen JC, Arnts H, van der Maarel SM, et al. Population-based incidence and prevalence of facioscapulohumeral dystrophy. Neurology 2014;83:1056-1059.

3. Tawil R. Facioscapulohumeral muscular dystrophy. Neurotherapeu tics 2008;5:601-606.

4. Sacconi S, Salviati L, Desnuelle C. Facioscapulohumeral muscular dystrophy. Biochim Biophys Acta 2014;4:607-614.

5. Padberg GW, Brouwer OF, de Keizer RJ, et al. On the significance of retinal vascular disease and hearing loss in facioscapulohumeral muscular dystrophy. Muscle Nerve 1995;2:S73-S80.

6. Brouwer OF, Padberg GW, Ruys CJ, et al. Hearing loss in facioscapulohumeral muscular dystrophy. Neurology 1991;41:18781881

7. Lutz $\mathrm{KL}$, Holte $\mathrm{L}$, Kliethermes $\mathrm{SA}$, et al. Clinical and genetic features of hearing loss in facioscapulohumeral muscular dystrophy. Neurology 2013;81:1374-1377.

8. Trevisan CP, Pastorello E, Tomelleri G, et al. Facioscapulohumeral muscular dystrophy: hearing loss and other atypical features of patients with large 4q35 deletions. Eur J Neurol 2008;15:13531358

9. van Deutekom JC, Wijmenga C, van Tienhoven EA, et al. FSHD associated DNA rearrangements are due to deletions of integra copies of a $3.2 \mathrm{~kb}$ tandemly repeated unit. Hum Mol Genet 1993; 2:2037-2042.

10. Wijmenga C, Frants RR, Brouwer OF, et al. Location of facioscapulohumeral muscular dystrophy gene on chromosome 4. Lancet 1990:336:651-653

11. Lemmers RJ, Tawil R, Petek LM, et al. Digenic inheritance of an SMCHD1 mutation and an FSHD-permissive D4Z4 allele causes facioscapulohumeral muscular dystrophy type 2. Nat Genet 2012; 44:1370-1374

12. Lemmers RJ, de Kievit P, Sandkuijl L, et al. Facioscapulohumeral muscular dystrophy is uniquely associated with one of the two variants of the $4 \mathrm{q}$ subtelomere. Nat Genet 2002;32:235-236.

13. Lemmers RJ, Wohlgemuth M, Frants RR, et al. Contractions of D4Z4 on 4qB subtelomeres do not cause facioscapulohumeral muscular dystrophy. Am J Hum Genet 2004;75:1124-1130.

14. Lemmers RJ, van der Vliet PJ, Klooster R, et al. A unifying genetic model for facioscapulohumeral muscular dystrophy. Science 2010 329:1650-1653.

15. Thomas NS, Wiseman K, Spurlock G, et al. A large patient study confirming that facioscapulohumeral muscular dystrophy (FSHD) disease expression is almost exclusively associated with an FSHD locus located on a 4qA-defined 4qter subtelomere. J Med Genet 2007;44:215-218.

16. Dixit M, Ansseau E, Tassin A, et al. DUX4, a candidate gene of facioscapulohumeral muscular dystrophy, encodes a transcriptional activator of PITX1. Proc Natl Acad Sci U S A 2007;104: 18157-18162. 
17. Snider L, Asawachaicharn A, Tyler AE, et al. RNA transcripts, miRNA-sized fragments and proteins produced from D4Z4 units: new candidates for the pathophysiology of facioscapulohumeral dystrophy. Hum Mol Genet 2009;18:2414-2430.

18. Vanderplanck C, Ansseau E, Charron S, et al. The FSHD atrophic myotube phenotype is caused by DUX4 expression. PLoS One 2011;6:e26820.

19. Tassin A, Laoudj-Chenivesse D, Vanderplanck C, et al. DUX4 expression in FSHD muscle cells: how could such a rare protein cause a myopathy? J Cell Mol Med 2012;17:76-89.

20. Geng LN, Yao Z, Snider L, et al. DUX4 activates germline genes, retroelements, and immune mediators: implications for facioscapulohumeral dystrophy. Dev Cell 2012;22:38-51.

21. Jones TI, Chen JC, Rahimov F, et al. Facioscapulohumeral muscular dystrophy family studies of DUX4 expression: evidence for disease modifiers and a quantitative model of pathogenesis. Hum Mol Genet 2012;21:4419-4430.

22. Broucqsault N, Morere J, Gaillard MC, et al. Dysregulation of 4q35- and muscle-specific genes in fetuses with a short D4Z4 array linked to facio-scapulo-humeral dystrophy. Hum Mol Genet 2013;22:4206-4214.

23. Ferreboeuf $M$, Mariot $V$, Bessieres B, et al. DUX4 and DUX4 downstream target genes are expressed in fetal FSHD muscles. Hum Mol Genet 2014;23:171-181.

24. Caruso N, Herberth B, Bartoli M, et al. Deregulation of the protocadherin gene FAT1 alters muscle shapes: implications for the pathogenesis of facioscapulohumeral dystrophy. PLoS Genet 2013;9:e1003550.

25. Sopko R, McNeill H. The skinny on Fat: an enormous cadherin that regulates cell adhesion, tissue growth, and planar cell polarity. Curr Opin Cell Biol 2009;21:717-723

26. Tanoue T, Takeichi M. Mammalian Fat1 cadherin regulates actin dynamics and cell-cell contact. J Cell Biol 2004;165:517-528.

27. Braun GS, Kretzler M, Heider $T$, et al. Differentially spliced isoforms of FAT1 are asymmetrically distributed within migrating cells. J Biol Chem 2007;282:22823-22833.

28. Gaillard MC, Roche S, Dion C, et al. Differential DNA methylation of the D4Z4 repeat in patients with FSHD and asymptomatic carriers. Neurology 2014;83:733-742.
29. Bustin SA, Benes V, Garson JA, et al. The MIQE guidelines: minimum information for publication of quantitative real-time PCR experiments. Clin Chem 2009;55:611-622.

30. Nagae S, Tanoue T, Takeichi M. Temporal and spatial expression profiles of the Fat3 protein, a giant cadherin molecule, during mouse development. Dev Dyn 2007;236:534-543.

31. Chen H. Atlas of genetic diagnosis and counseling. Totowa, NJ: Humana Press, 2006

32. Krom YD, Dumonceaux J, Mamchaoui K, et al. Generation of isogenic D4Z4 contracted and noncontracted immortal muscle cell clones from a mosaic patient: a cellular model for FSHD. Am J Pathol 2012;181:1387-1401.

33. Snider L, Geng LN, Lemmers RJ, et al. Facioscapulohumeral dystrophy: incomplete suppression of a retrotransposed gene. PLoS Genet 2010;6:e1001181.

34. Yao Z, Snider L, Balog J, et al. DUX4-induced gene expression is the major molecular signature in FSHD skeletal muscle. Hum Mol Genet 2014;23:5342-5352

35. Young JM, Whiddon JL, Yao Z, et al. DUX4 binding to retroelements creates promoters that are active in FSHD muscle and testis. PLoS Genet 2014;9:e1003947.

36. Puppo F, Dionnet E, Gaillard MC, et al. Identification of variants in the $4 \mathrm{q} 35$ gene FAT1 in patients with a facioscapulohumeral dystrophy-like phenotype. Hum Mutat. 2015;36:443-453.

37. Ciani L, Patel A, Allen ND, ffrench-Constant C. Mice lacking the giant protocadherin mFAT1 exhibit renal slit junction abnormalities and a partially penetrant cyclopia and anophthalmia phenotype. Mol Cell Biol 2003;23:3575-3582.

38. Mahoney PA, Weber $U$, Onofrechuk $P$, et al. The fat tumor suppressor gene in Drosophila encodes a novel member of the cadherin gene superfamily. Cell 1991;67:853-868.

39. Scionti I, Fabbri G, Fiorillo C, et al. Facioscapulohumeral muscular dystrophy: new insights from compound heterozygotes and implication for prenatal genetic counselling. J Med Genet 2012;49:171-178.

40. Cabianca DS, Casa V, Bodega B, et al. A long ncRNA links copy number variation to a polycomb/trithorax epigenetic switch in FSHD muscular dystrophy. Cell 2012;149:819-831.

41. Tanoue T, Takeichi M. New insights into Fat cadherins. J Cell Sci 2005:118:2347-2353. 